\title{
Recent Advances in Carbon Dots
}

\author{
Theodoros Chatzimitakos $(\mathbb{D})$ and Constantine Stalikas * \\ Laboratory of Analytical Chemistry, Department of Chemistry, University of Ioannina, 45110 Ioannina, Greece \\ * Correspondence: cstalika@uoi.gr; Tel.: +30-265-100-8414
}

Received: 11 July 2019; Accepted: 16 July 2019; Published: 22 July 2019

It is unequivocally true that in recent years, carbon-based nanomaterials such as graphene, carbon nanotubes, and fullerenes, have attracted tremendous interest and an increasing number of applications have reaped their numerous benefits. Lately, carbon nanodots (CNDs) have emerged as a new class of carbon-based nanomaterials, which have "outshone" other carbon-based nanomaterials, as they possess a unique combination of outstanding, enthralling, and favorable properties for many applications. They are the only carbon nanomaterials with inherent photoluminescence whereby multitudinous applications have been developed.

CNDs have come into their own as a standalone research field, but their full potential is yet to be revealed and harnessed. Nowadays, CNDs are utilized as probes for analytical or biological applications; bioimaging agents for live cell and animal imaging purposes; drug carriers; and photosensitizers in photodynamic therapy as well as in photocatalysis, solar cells, optronics, etc.

To better understand their properties and gain deeper knowledge on the subject, concise research and review articles are needed. To address this need, we launched a Special Issue of $C$-Journal of Carbon Research, "Recent Advances in Carbon Dots", which is dedicated to the advancement of this novel field. In this Issue, Sciortino et al. study the emission characteristics of N-doped CNDs (from microwave treatment of citric acid and urea) in an effort to better understand the fluorescence emission mechanism of CNDs using various solvents [1]. The study was conducted at two different temperatures (300 and $80 \mathrm{~K}$ ) and two different solvents (one polar and one apolar). Interestingly, the authors found that the excitation-emission Stokes shift in the polar solvent is due to solvent relaxation and that the emission shape of CNDs is due to static inhomogeneity. According to the authors, "These results may pave the way to strategies capable of selectively enhancing or suppressing the tunability of CD fluorescence, which may be useful for several applications". In another study, Koutsioukis et al. described the use of CNDs to prepare water-based CNDs ink for gravure painting [2]. CNDs prepared by citric acid and triethylenetetramine were mixed with a commercially available varnish to prepare the ink. The ink was found to be highly appropriate for printing applications, since the printed structures were stable according to ISO 2836/2004. This study is the first of its kind to highlight the potential of CNDs for fluorescent gravure printing inks. Only a few studies have been reported up to now that have used CNDs to prepare fluorescence inks for anti-counterfeiting applications.

In his extensive review, Ganeshlenin Kardasamy summarizes the recent developments in doped and co-doped CNDs, with respect to the various heteroatoms, the synthesis method, and parameters and the quantum yield of the CNDs [3]. In addition to the above, multiple applications of the CNDs are discussed, including their use in light-emitting diodes, solar cells, anti-counterfeiting applications, and optical sensors for the detection of metal ions, drugs, and pesticides, as well as their use for gene delivery and temperature probing. On the basis of the utilization of CNDs in biological-related applications, two more review articles were published. Lin et al., who focused on the use of CNDs for sensing and killing microorganisms [4], presented valuable data regarding the synthesis, functionalization, and toxicity profiles of many synthesized CNDs, making it easier to understand their antimicrobial properties. Their review focused not only on bacteria but also on fungi and viruses. They discussed the mechanisms via which sensing and killing of microorganisms are 
achieved. Kasouni et al. presented a comprehensive review that extensively discusses the use of CNDs in bioimaging applications [5]. In this article, multiple applications of CNDs as imaging agents for prokaryotes, eukaryotes, and animals are provided and discussed. In this context, multiple "unique" and more sophisticated bioimaging-based applications are highlighted. Typical examples are the use of CNDs for selective imaging of certain cells over others (e.g., deciphering of Gram-positive from Gram-negative bacteria); the selective imaging of cancer cells or tissue over non-cancerous cells or normal tissue; and the biosensing applications that make feasible the real-time monitoring of specific molecules inside cells, so that conclusions for abnormal conditions can be drawn.

After a careful reading of this Special Issue, it becomes evident that CNDs constitute a fruitful research area, and many other applications are yet to be developed. As Guest Editors of this Special Issue, we would like to thank all authors for their excellent contributions, and we hope that the Special Issue meets the expectations of the readers.

Conflicts of Interest: The authors declare no conflict of interest.

\section{References}

1. Sciortino, A.; Cannas, M.; Messina, F. Temperature-dependence of solvent-induced stokes shift and fluorescence tunability in carbon nanodots. C 2019, 5, 20. [CrossRef]

2. Koutsioukis, A.; Belessi, V.; Georgakilas, V. Fluorescent carbon dots ink for gravure printing. C 2019, 5, 12. [CrossRef]

3. Kandasamy, G. Recent advancements in doped/co-doped carbon quantum dots for multi-potential applications. C 2019, 5, 24. [CrossRef]

4. Lin, F.; Bao, Y.W.; Wu, F.G. Carbon dots for sensing and killing microorganisms. C 2019, 5, 33. [CrossRef]

5. Kasouni, A.; Chatzimitakos, T.; Stalikas, C. Bioimaging applications of carbon nanodots: a review. C 2019, 5, 19. [CrossRef]

(C) 2019 by the authors. Licensee MDPI, Basel, Switzerland. This article is an open access article distributed under the terms and conditions of the Creative Commons Attribution (CC BY) license (http://creativecommons.org/licenses/by/4.0/). 